\title{
Pierre VADEBONCOEUR [1920-2010]
}

avocat, syndicaliste et écrivain québécois.

(1991)

\section{"Pour une dynamique de notre culture."}

Un document produit en version numérique par Réjeanne Toussaint, ouvrière bénévole, Chomedey, Ville Laval, Québec

Page web personnelle. Courriel: rtoussaint@aei.ca

Dans le cadre de la collection: "Les classiques des sciences sociales"

Site web: http://classiques.uqac.ca/

Une bibliothèque fondée et dirigée par Jean-Marie Tremblay, sociologue

Une collection développée en collaboration avec la Bibliothèque Paul-Émile-Boulet de l'Université du Québec à Chicoutimi

Site web: http://bibliotheque.uqac.ca/ 


\section{Politique d'utilisation de la bibliothèque des Classiques}

Toute reproduction et rediffusion de nos fichiers est interdite, même avec la mention de leur provenance, sans l'autorisation formelle, écrite, du fondateur des Classiques des sciences sociales, Jean-Marie Tremblay, sociologue.

Les fichiers des Classiques des sciences sociales ne peuvent sans autorisation formelle:

- être hébergés (en fichier ou page web, en totalité ou en partie) sur un serveur autre que celui des Classiques.

- servir de base de travail à un autre fichier modifié ensuite par tout autre moyen (couleur, police, mise en page, extraits, support, etc...),

Les fichiers (.html, .doc, .pdf., .rtf, .jpg, .gif) disponibles sur le site Les Classiques des sciences sociales sont la propriété des Classiques des sciences sociales, un organisme à but non lucratif composé exclusivement de bénévoles.

Ils sont disponibles pour une utilisation intellectuelle et personnelle et, en aucun cas, commerciale. Toute utilisation à des fins commerciales des fichiers sur ce site est strictement interdite et toute rediffusion est également strictement interdite.

L'accès à notre travail est libre et gratuit à tous les utilisateurs. C'est notre mission.

Jean-Marie Tremblay, sociologue

Fondateur et Président-directeur général, LES CLASSIQUES DES SCIENCES SOCIALES. 
Cette édition électronique a été réalisée par Réjeanne Toussaint, bénévole, Courriel: rtoussaint@aei.ca

à partir de :

\section{Pierre VADEBONCOEUR}

\section{"Pour une dynamique de notre culture."}

Un texte publié dans l'ouvrage d'Yvan Lamonde, avec la collaboration de Gérard Pelletier, CITÉ LIBRE. Une anthologie, pp. 228235. Montréal : Les internationales Alain Stanké, 1991, 415 pp.

[Autorisation formelle accordée par Monsieur Yvan Lamonde et son éditeur, Les Éditions Hurtubise HMH ltée, le 2 septembre 2008 de diffuser ce livre dans Les Classiques des sciences sociales.]

\section{dif Courriel : yvan.lamonde@mcgill.ca}

Polices de caractères utilisée :

Pour le texte: Times New Roman, 14 points.

Pour les citations : Times New Roman, 12 points.

Pour les notes de bas de page : Times New Roman, 12 points.

Édition électronique réalisée avec le traitement de textes Microsoft Word 2008 pour Macintosh.

Mise en page sur papier format : LETTRE US, 8.5'” x 11'”.

Édition numérique réalisée le 21 mars 2013 à Chicoutimi, Ville de Saguenay, Québec.

\section{f Fait avec}




\section{Pierre VADEBONCOEUR [1920-2010]}

avocat, syndicaliste et écrivain québécois.

\section{"Pour une dynamique de notre culture."}

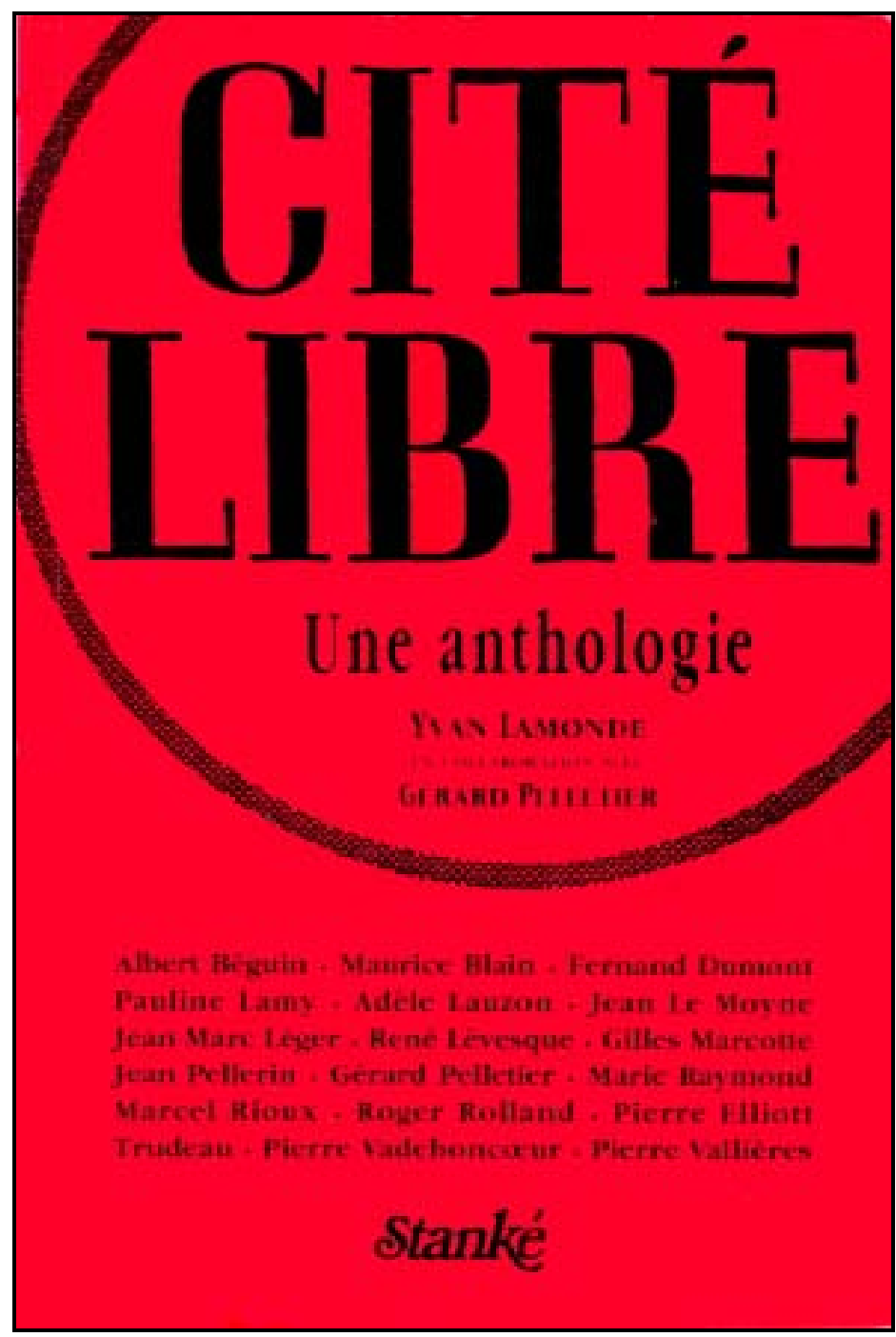

Un texte publié dans l'ouvrage d'Yvan Lamonde, avec la collaboration de Gérard Pelletier, CITÉ LIBRE. Une anthologie, pp. 228-235. Montréal : Les internationales Alain Stanké, 1991, 415 pp. 
Vadeboncoeur, Pierre (1920-). Syndicaliste, essayiste. Licencié en droit (1943), il s'implique dans la grève de l'amiante et joint alors le syndicalisme (CTCC-CSN). Collabore à Liberté, Socialisme, Parti pris, Maintenant. Parmi ses essais, mentionnons: La Ligne du risque (1963), L'Autorité du peuple (1965), La Dernière heure et la première (1970), Indépendances (1972), Essais inactuels (1987). 
CITÉ LIBRE. Une anthologie.

VI. Sur l'identité

\section{"Pour une dynamique de notre culture."}

par Pierre Vadeboncoeur

Pierre Vadeboncoeur. « Pour une dynamique de notre culture », Cité libre, 2, 1-2 (juin-juillet 1952) : 12-19.

Un texte publié dans l'ouvrage d'Yvan Lamonde, avec la collaboration de Gérard Pelletier, CITÉ LIBRE. Une anthologie, pp. 228235. Montréal : Les Éditions internationales Alain Stanké, 1991, 415 pp. [Autorisation formelle accordée par Yvan Lamonde et son éditeur, le 2 septembre 2008 de diffuser ce livre dans Les Classiques des sciences sociales.]

Sans cesse rappelé à la conscience de soi par une philosophie pleine de soucis d'ordre culturel, notre peuple périclite pourtant dangereusement dans sa culture.

Cette aberration historique progressive est inconsciente parce qu'inconsciente est l'erreur philosophique qui en est la cause. Elle est soustraite au regard notamment par la couverture même des enseignements dispensés, c'est-à-dire par l'illusion de l'idéalisme. 
La décadence dont il est ici question, ce n'est pas celle, banale, que l'on doit imputer à des causes extérieures, par exemple aux facteurs de contamination par l'étranger ; non, il s'agit d'une erreur d'intelligence et de vie, et d'une très grave erreur d'enseignement, que l'on peut préciser ainsi : la culture a cessé de savoir où se trouve l'homme réel, quelles sont ses puissances et quoi lui révéler de désirs et de forces sous les espèces de l'idéal ; notre culture a totalement négligé de fouetter les puissances de l'homme.

Mais de quelles puissances s'agit-il ? Répondre directement à cette question serait risquer de le faire en termes abstraits. Il vaut mieux signaler quelques déficiences, qui situent mieux que les définitions les problèmes de la culture : la faiblesse de nos élans politiques, leur rareté, notre peu d'ambition, notre honteuse indifférence à l'égard de l'ignorance, notre laisser-aller, notre paresse critique à l'égard des institutions, notre conformisme idéologique, notre indiscipline personnelle, notre peu de force offensive (par exemple : sur le plan personnel, pour réaliser une oeuvre), notre complexe juvénile à l'égard de l'autorité et, corollairement, notre manque foncier d'indépendance et partant de conscience et de virilité ; notre amour d'un traditionalisme absolument insupportable pour l'esprit ; notre consentement universel à vivre dans l'imperfection de foi ; notre bénignité ; notre docilité, etc.

Notre culture est construite d'une part sur une extraordinaire indifférence de regard pour ces réalités et, d'autre part, sur la présentation d'une philosophie de haute tradition, soi-disant fondée sur la " connaissance de l'homme », étiquette notoire de toute une science constamment débordée par l'investigation moderne. Nous sommes un peuple qui, par l'idée, a singulièrement désappris l'homme.

Mais indiquons par des observations un peu plus immédiates le vaste mécompte d'une philosophie indigène qui, prétendant enseigner la culture, ne connaît point que chez nous la culture est dans une condition mortelle.

L'impasse de notre culture n'a guère fait jusqu'ici l'objet de notations critiques que sur des points d'importance seconde et de l'ordre 
des conséquences, comme la pénurie d'oeuvres littéraires ou nos échecs en politique nationaliste. Du reste, ces observations étaient généralement de simples expressions de regrets. La littérature morale et politique, dans ses oeuvres maîtresses, omettait de remettre en question notre culture ; elle ne la regardait pas dans ce qu'elle est devenue, mais dans ses sources. Nous nous sommes engagés, depuis cent ans, dans une baisse culturelle dont les lettres, même littéraires, ne rendent pas le compte le moindrement adéquat, bien que cette crise équivaille à un échec historique. Les ouvrages traitant de notre situation font une grande place à la conjoncture extérieure, aux difficultés de notre économie, par exemple; mais l'âme du peuple, et plus encore celle de l'individu, son degré actuel d'énergie, de courage, d'indépendance, d'intolérance de l'injustice, de possession de soi, de liberté, d'usage de la liberté, de hardiesse intellectuelle, de personnalisme, d'autonomie intérieure, de volonté, de force d'âme, de sens de la conquête, de passion, ne font pas l'objet d'analyses et surtout ne fournissent pas le sujet de mouvements idéologiques majeurs. On ne se préoccupe pas davantage de capter les courants historiques ou idéologiques qui pourraient contribuer à nous libérer. On nous a enseigné l'histoire profane et la morale de l'Église ; mais autant dégageait-on de leçons claires de ces études, autant le problème de culture de tout un peuple, lié non pas à son idéologie ou à son passé, mais à ses réalités et particulièrement à ses réalités intérieures, demeurait-il sans solution. Non seulement demeurait-il sans réponse, mais c'est avec stupéfaction que l'on constate qu'il n'est même pas posé. La littérature n'a pas examiné notre problème moral ; elle s'est contentée de formuler l'idéal omnivalent du salut, ce qui naturellement ne relève pas de l'observation et ne fournit pas non plus de soi les représentations neuves, multiformes, énergétiques, dont la culture a tant besoin.

Notre enseignement moral, politique, journalistique, universitaire, est à caractère nettement essentialiste. Indépendamment de ce qu'a pu nous apprendre la philosophie contemporaine sur l'existentialisme et sur d'autres sagesses qui ont rompu à des degrés divers avec ce que l'essentialisme peut comporter de tyrannique, il reste que, sur un plan 
purement empirique, le fait suivant appelle l'attention : notre culture se pose les problèmes de la loi (le nom même du journal le plus représentatif de notre pensée est Le Devoir), mais ceux de l'existence lui sont à peu près étrangers, c'est-à-dire : tout ce qui importe en deçà de la loi, en deçà du bien et du mal, en nature, en vitalité, en vertus si l'on peut dire autonomes, en qualités traduites par les littératures, en capacités qui font la vigueur fondamentale du type humain. Et de fait, nous accusons une déficience notable en cette nature, cette vitalité, ces vertus, ces qualités, ces capacités. Ces choses ne sont peut-être pas indifférentes à nos jugements individuels, mais elles le sont assurément à notre culture. Notre culture comporte très peu d'impératifs antémoraux, bien que le rôle en soit immense et qu'aucune société ne puisse s'élever vraiment dans l'échelle des êtres collectifs sans les puissantes suggestions d'un idéal à hautes doses d'appels directs à l'existence.

Il est pourtant un fait curieux : c'est que les propos sur notre délabrement, disons psychologique, courent les rues ; ils abondent dans les conversations privées. Mais, chose dès lors plus singulière encore, les oeuvres ne saisissent pas cette matière de réflexions. L'observation de nos auteurs s'est exercée sur les situations (école historique, économique) et leur sagacité sur des problèmes de scolastique (école philosophique traditionnelle).

Explications, leçons, alarmes, ne se rapportaient point à la psychologie nationale, à l'âme actuelle, qu'il se fût agi pourtant d'entraîner et d'assaillir de multiples façons notamment par l'ironie. Peuple qui n'a point eu d'ennemis intellectuels. Petit classicisme qui n'a pas eu d'adversaires spirituels. Point de maîtres, non plus, qui aient, d'enseignement ou d'exemple, renouvelé le coeur. Ce peuple est en rupture de tout savoir des enseignements divins ou profanes qui recommandent la violence et qui de là promettent la possession. Nous sommes sans maîtres qui nous eussent parlé de l'âme et de ses énergies extrêmes, et nous sommes sans culture qui nous mettrait, par la seule ambiance, à une exaltante proximité des biens surnaturels, temporels, intellectuels, des biens de poésie, des biens de l'action, des biens du vivre, enfin. Nous sommes sans critique historique de l'âme nationale et une vivan- 
te philosophie nous manque à tous égards, quoi que puisse laisser croire notre formulation philosophique traditionnelle.

En un vague rapport avec la décadence de la culture nationale se sont bien élevées une certaine littérature militante et une affirmation philosophique, il est vrai, mais elles n'en tiennent pas compte comme telle et ne la rencontrent pas sur le terrain dialectique. Si bien que notre pensée est à cet égard purement symptomatique. Elle dérive indirectement de notre crise culturelle, qu'elle sent obscurément, sans toutefois fournir réponse. Elle exprime un malaise, une inquiétude, un sentiment de l'erreur et de l'impasse, qu'elle traduit, indirectement, maladroitement, d'une manière substituée, par habitude, par inconscience, sur les thèmes de la pensée nationaliste. C'est un indice très visible de notre insuffisance dialectique que de voir une crise culturelle s'exprimer, sur le plan de la réponse théorique, par le regonflement incessant des mêmes idéaux ${ }^{1}$. À toute crise, même économique, répond chez nous la recristallisation automatique d'un système à peu près invariable. Rien d'étonnant : dans un pays où la tradition est sacrée et la pensée rare, c'est la tradition que l'on charge de représenter à toutes fins le réel. C'est elle qu'on a sous la main et c'est par ailleurs elle qui, de son chef, se proclame l'image de la réalité et l'arme du bien. Une répétition indéfinie des mêmes propositions, indépendamment de toutes conditions nouvelles, dans l'ignorance culturelle de ces

1 Le nationalisme, chez nous, est une faible ébauche de mouvement vers le pouvoir. Il ne convoite cependant pas le pouvoir ; il existe comme un pouvoir abstrait et méditatif en marge du pouvoir réel et actif, et chacune de ses recrudescences représente un mouvement instinctif, mais aveugle et tout à fait irrésolu vers le pouvoir. État abstrait dans l'État réel, ce qu'il cherche, ce n'est pas la réponse réelle aux problèmes, mais la réalisation, d'ailleurs négligée, de ce qu'il est le plus profondément lui-même : un État préfabriqué, que le pouvoir réaliserait dans les faits, tel qu'il est, avec sa philosophie et ses formes de toujours ; et il croit que bien des problèmes se régleraient par son accession au pouvoir. Ce bizarre phénomène politique est d'autant mieux explicable que nous demeurons fort peu accessibles à la pensée, laquelle briserait sans doute ces formes trop conservées. Aux problèmes, nous répondrons par un État, et cet État n'a même pas la vigueur de se réaliser. Ce phénomène n'est pas pour peu dans la fixation de l'appareil idéologique qui sous-tend nos quelconques projets politiques, ni dans l'arrêt de notre pensée tout court. 
conditions, à distance de toute chance d'une philosophie nouvelle, est donc à prévoir à partir de là dans ce cercle. Dans une telle conjoncture, nous assistons au déballage périodique et obstiné de ce qui, aux termes de la tradition, est toujours considéré comme le remède, comme la solution, suffisante, complète, et que seule, paraît-il, la malice des hommes empêche de prévaloir pour produire ses bienfaisants effets. (Un pareil point de vue identifiait la pensée des catholiques européens, vers 1848, en présence de l'esclavage industriel, comme nous l'observerons plus loin.) (La malice des hommes est un des sujets de méditation les plus essentiels, les plus intimement implicites de la pensée nationaliste: il serait pourtant plus juste de concevoir que l'égoïsme humain - les nationalistes se rendent-ils compte à quel point ils y pensent ? - n'est pas tant en cause que leur philosophie de l'action, leurs buts, leur inspiration, leur formation culturelle et leurs méthodes.)

Littérature militante et positions philosophiques réaffirment à toute force les principes et les buts traditionnels. C'est que l'on sent bien que la décadence menace, entre autres choses, les formes héritées de culture. Notre volonté de maintenir ces formes nous fait aisément nous représenter cette décadence comme l'ennemie spécifique des idéaux traditionnels. Cela provoque une défense spécifique des formes classiques, une défense spécifique par ces formes. La décadence morale (et culturelle) est combattue par l'énoncé insistant de la morale reçue. Ce que la culture traditionaliste appréhende de la décadence, ce sont ses manifestations contraires à la morale traditionnelle : impossible en effet pour l'Église de ne pas constater le matérialisme grandissant, les sept péchés capitaux et tout ce qui offense son enseignement. Mais justement, c'est en tant que la morale reconnue subit une atteinte que des protestations s'élèvent et que l'on émet des directives. C'est alors la prédication traditionnelle qui s'ébranle. L'atteinte même l'exaspère et la valorise exclusivement. La crise de culture est interprétée sur le plan individuel et comme la conséquence d'une infidélité à des règles connues et codifiées ; sous la juridiction, par conséquent, d'une prédication classique selon ces règles. Elle ne pose donc pas de problème 
neuf et spécifique au jugement traditionaliste. Cela fait qu'une civilisation entière peut lentement se dégrader sans que la pensée consente à voir le mal ailleurs que sur un plan où l'on puisse faire référence aux normes classiques. D'où l'anachronisme et la réaction. D'où le fait signalé plus haut : nul n'a décrit notre crise de culture ; nul n'en a saisi dramatiquement l'incidence historique en marge de nos intérêts idéologiques traditionnels. Ceux-ci répondent en leur propre nom, et c'est tout. Ils n'ont aucune imagination.

Un parallélisme frappant au phénomène que je viens de décrire existe dans le domaine proprement national et politique. Là encore, la décadence émeut des énergies. Le nationaliste déplore des faits et des états qui sont bien de nature à le frapper : l'inertie politique du peuple, qui lui fait rater ses campagnes électorales, par exemple, cela lui saute aux yeux. Mais cette constatation tourne au profit de la prédication traditionnelle de l'idéal nationaliste, et il repart en guerre pour son système. Ici encore, une authentique observation du réel subit l'annexion au bénéfice d'une pensée faite d'avance. S'il a remarqué l'inertie populaire, cela, par exemple, ne le conduit pas à la révolte contre la culture régnante, ni même à l'examen critique de cette dernière, mais au contraire à l'affirmation en vrac et redoublée de celle-ci et à la confirmation de tout l'édifice qui la conserve. Les déclins culturels ne nous poussent pas à discuter mais à réaffirmer les objectifs de la tradition. Un phénomène de cette nature se produisit en Europe vers le milieu du siècle dernier à propos de l'industrialisme et de la réduction des masses en esclavage : la chrétienté poursuivait un enseignement de justice et de charité excellent en principe mais périmé dans ses méthodes, tandis que s'imposait une action carrément révolutionnaire. Bref, les défenses qu'on oppose aux conditions régnantes s'exercent dans le sens des lieux communs. C'est ce qui fait qu'une certaine technique de la résistance paraisse suffire à une collectivité que l'on croit par ailleurs assez armée sur le plan des idées.

Voici en particulier un petit exemple qui en dit long sur ce qu'on pourrait appeler le subconscient idéologique de notre milieu : la fondation des Jeune-Canada en pleine crise économique; mouvement 
dont la signification était avant tout nationaliste et qui fut très préoccupé des questions de langue et de nationalité : problèmes intempestifs, s'il en fut à cette époque-là. Le sens de cet épisode s'avère plus criant encore si l'on rappelle son retentissement et le souvenir qu'il a laissé par comparaison à certain mouvement, fondé vers le même temps mais saisi des véritables problèmes de l'époque, et aujourd'hui complètement oublié. Mais à quelle protestation véhémente, autre qu'athée et communiste, la crise économique donna-t-elle lieu ? Nous pouvons juger du peu que nous réalisâmes alors pour la culture au peu de ce qui est resté, dans le lyrisme politique, dans le lyrisme populaire, de cette période d'infortune publique et à la réapparition naïve, en corollaire, durant ces temps terribles, des thèmes intacts de la pensée nationaliste rebattue du XIXe siècle. Nous n'interprétâmes alors aucunement la misère universelle et l'insupportable épreuve de notre propre ville. Nous manquions à ce point de lecture et de violence, d'observation et de pathétique, que les malheurs de la crise ne suffirent point à nous pousser à contre-affirmer dans nos frontières, avec la passion qui s'imposait, l'injustice d'un système économique qui ne s'était jusqu'alors pleinement caractérisé que par l'oppression qu'il avait fait peser ailleurs sur une vaste partie du globe.

Dans la même perspective, on peut trouver qu'au temps actuel, au carrefour économico-politico-philosophique du monde, la pensée traditionnelle, du moins chez nous, fait une lourde concurrence à toute introduction d'une pensée neuve, comme ce fut apparemment le cas durant la crise. Elle draine une somme considérable d'intentions et opère une diversion idéologique telle qu'il paraît peu probable que le mouvement révolutionnaire, qui s'impose par l'univers entier, puisse s'amorcer ici ; et non seulement ce mouvement, mais aussi les approches modernes du problème de la culture.

Cependant par quels indices sommes-nous amenés à nous poser le problème de la culture, quand notre héritage doctrinal demeure si élevé, quand notre histoire semble nous poser surtout des problèmes économiques, et quand, au surplus, presque personne dans nos classes dirigeantes ne semble s'être inquiété de questions dont l'expression 
n'apparaît pas dans nos doctrines et que ne suggèrent pas les situations extérieures classiques?

Ces indices, comme je l'ai déjà laissé entendre, sont d'ordre psychologique. La culture d'un peuple, c'est, avant tout, si l'on peut dire, son état psychologique. La culture s'exprime essentiellement par un état psychologique qui en est la résultante et qui en est aussi le barème : on la juge à la psychologie qu'elle détermine. C'est pourtant là un point de vue qui ne nous est pas coutumier. L'étranger, lui, et même notre compatriote anglo-saxon, nous jugent sur cette mesure. Ils s'étonnent, par exemple, de tel ou tel trait de docilité juvénile, ou de passivité ; puis ils regardent notre pensée, pour la critiquer. Mais quant à nous, s'il est vrai que nous nous arrêtons quelquefois à deviser sur nos lacunes de caractère, il ne nous apparaît guère que nos observations mettent notre culture en cause.

Il s'agit en effet de savoir ce que notre pensée a fait de nous. Il s'agit de voir le type d'hommes qu'elle a créé. Ce qu'on regarde d'abord d'un tel point de vue, ce n'est plus la doctrine ou l'idéal d'une collectivité, mais ses réactions primaires, son comportement direct, la réponse décisive de son être noble ou vil, fier ou honteux, sceptique ou assuré, sa musculature, ses dominantes pour le succès ou pour l'échec, la vigueur et la promptitude de son effort, son mépris des obstacles ou sa timidité. Coup d'œil direct, vision de l'existence même. Nous n'avons pas ce coup d'oeil. Notre culture est même caractérisée par l'absence de ce regard volontaire de cette intuition réaliste, de cette notion de l'homme absolument dépouillée, pour l'instant, de toute superstructure.

D'où vient que, si révélateur et si riche de critique révolutionnaire en puissance, ce point de vue, indispensable à toute pensée vivante, nous soit étranger? Comment concevoir que tout un peuple pense sans même regarder la pierre de touche de sa pensée : sa propre âme ? C'est peut-être que beaucoup d'idées, de projets, de méthodes et d'institutions sont menacés par le redressement qui s'ensuivrait. 
Tout se passe comme si, à la faveur de la présence abstraite de notre idéologie, nous nous fussions oubliés nous-mêmes et que notre histoire, se croyant préservée par la doctrine, se fût déroulée dans la plus parfaite indifférence de ce qu'il pouvait advenir de notre culture réelle, concrète. Nous avons notamment méconnu ce qu'il advient de la capacité d'un peuple qui croit pouvoir être éduqué sans de fortes images de la valeur profane.

Nous languissons d'une maladie mortelle du caractère. Or, la réalité culturelle présente d'un peuple en rapide déclin psychologique et moral ne doit plus être masquée par le culte mal à propos de ce qu'il traînasse comme idéal. Sa réalité culturelle actuelle doit être la première image que l'on se fasse de lui. Elle doit donner de singulières alarmes comme provoquer des résolutions en rapport avec le problème de créer des hommes et des choses dans un contexte historique qui se refuse à y aider.

Notre culture à venir, que l'on cherche à assurer par des mots d'ordre de fidélité au passé, par une sagesse placide, précautionneuse, et par des formules usées jusqu'à la corde, dépend strictement d'une sorte de révolution psychologique.

Toute philosophie morale, tout essai de transmutation des valeurs, toute tentative d'action sur le pouvoir civilisateur et réalisateur d'un peuple, est un effort pour influencer sa psychologie ou pour changer les conditions qui influencent celle-ci. Ce seul aperçu suffit à laisser entrevoir l'importance d'un point de vue qui s'inquiète des réactions caractérielles d'une société, de sa réponse psychologique ; importance particulièrement évidente pour une pensée collective toute entière réfugiée dans l'idée qu'il faut sauver certaines idées.

Sans doute, de multiples nécessités sont, de leur nature, antérieures à un idéal culturel nouveau, et il ne faut pas perdre de vue le rang dialectique du problème culturel. Il est par suite indispensable de comprendre qu'une révolution psychologique dépend elle-même d'une étonnante révolution dans les entreprises, et d'orientations historiques nouvelles, en accord avec la dynamique du monde contemporain et 
même précédant celui-ci, duquel on ne prendra jamais une vue assez audacieuse. Le problème culturel dépend aussi de l'introduction, dans notre pensée, d'une liberté inconnue jusqu'ici, et de plusieurs autres facteurs. Il est sous l'empire de conditions politiques, économiques et techniques redoutables; et, de cet angle, notre situation donne peu à espérer. Mais il se traite aussi à son mérite, et une culture contrainte, rétrécie, contenue, brimée, offre mieux qu'une autre, d'une manière isolable, son problème spécifique. Plus qu'une autre qui aurait atteint et dépassé ses dimensions dans tous les sens et qui souffrirait d'âge, de raffinement et de surabondance, elle est susceptible d'une influence libératrice.

Il faut être affamé d'existence. Notre culture réclame par-dessus tout cet impératif. 